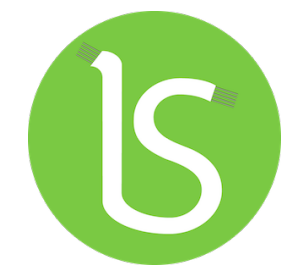

\title{
Propuesta metodológica de implementación de las Tecnologías de la Información para incrementar la competitividad de las MiPymes del sector económico comercial
}

\author{
Luis Alejandro Gazca Herrera \\ Enrique Victoria Marurib \\ Sergio Ignacio Parra Salmerónc
}

Resumen - Las Micro, Pequeñas y Medianas Empresas (MiPymes), impulsan la economía en el ámbito global, nacional, regional y local. En México, coexisten un gran número de MiPymes, lo anterior, de acuerdo con el número de trabajadores; en ellas se concentran casi la tercera parte del personal ocupado total del país y más de la mitad del Producto Interno Bruto (PIB) nacional; dejando un número muy reducido en comparativa con los grandes establecimientos. Pese a lo anterior, el nivel de competitividad que muestran las MiPymes señaladas resulta demasiado bajo, derivado de factores tales como la falta de implementación de Tecnologías de Información (TI) en sus actividades clave, que, en consecuencia, limita el incremento de la competitividad en el mercado en el que realizan su actividad comercial. El análisis situacional en el ámbito local no es prolífico, por lo que existe la necesidad de realizar el estudio de las condiciones en que operan las $v$ comerciales respecto de la integración de TI, por lo que el objetivo del presente será proponer un método de seis pasos de implementación de TI en MiPymes comerciales en Xalapa, Ver., México, con base en sus diversas necesidades, $y$ de esa manera, incrementar su competitividad empresarial.

Palabras clave - Competitividad, Tecnologías de Información, Economía, Sector Comercial.

Abstract - Micro, Small and Medium Enterprises (MSMEs), drive the economy at the

CÓMO CITAR

HOW TO CITE:

Gazca Herrera, L. A., Victoria

Maruri, E., y Parra Salmerón, S.

I. (2022). Propuesta

metodológica de

implementación de las

tecnologías de la información

para incrementar la

competitividad de las MiPymes

del sector económico

comercial. Interconectando

Saberes, (13), 19-36.

https://doi.org/ 10.25009/is.v0il 3

.2725

Recibido: 10 de agosto de 2021 Aceptado: 22 de noviembre de 2021

Publicado: 31 de enero de 2022 global, national, regional, and local levels. In Mexico, many MSMEs coexist, the above, according to the number of workers; Almost a third of the country's total employed personnel and more than half of the national Gross Domestic Product (GDP) are concentrated in them; leaving a very small number in comparison with the large establishments. Despite the above, the level of competitiveness shown by the mentioned MSMEs is too low, derived from factors such as the lack of implementation of Information Technology (IT) in their key activities, which consequently limits the increase in competitiveness in the market in which you do business. The situational analysis at the local level is not prolific, so there is a need to study the conditions in which commercial MSMEs operate concerning IT integration, so the objective of this document will be to propose a method of six IT implementation steps in commercial MSMEs in Xalapa, Ver., Mexico, based on their diverse needs, and thus increase their business competitiveness.

Keywords - Competitiveness, Information Technologies, Economy, Commercial Sector.

${ }^{a}$ Universidad Veracruzana, México. E-mail: Igazca@uv.mx

${ }^{b}$ Universidad Veracruzana, México. E-mail: evictoria@uv.mx

c Universidad Veracruzana, México. E-mail: sergio p sal@hotmail.com 


\section{INTRODUCCIÓN}

El conjunto de factores que inciden en la competitividad de las empresas se relaciona entre sí para fortalecer la estructura, favorecer el ejercicio óptimo de los recursos y obtener de esta manera, una posición preponderante dentro del mercado en el que se desenvuelven. Hoy en día, en el contexto global, la puesta en marcha de empresas con diferentes perfiles es considerado un detonante de la competitividad de un país, pues permiten activar intrínseca y extrínsecamente los factores de producción de acuerdo con las actividades que el sector en el que se desenvuelven les exija. Para el caso de esta investigación, el enfoque recae en el sector económico comercial, mismo que se estratifica de acuerdo con ciertos aspectos, los cuales pueden ser diferentes según la región geográfica, sin embargo, se centran tanto en el personal empleado como en la facturación anual. Esta estratificación permite separar en cuatro grupos o tamaños a las empresas, las cuales consideramos como micro, pequeñas, medianas y grandes, sin embargo, para la presente, se considerará el segmento conformado por las tres primeras: Micro, Pequeñas y Medianas empresas (MiPymes), soslayando a las grandes empresas. La importancia económica de las MiPymes se logra apreciar gracias al número de establecimientos y personal ocupado en el escenario global nacional y local, en México, según los Resultados Definitivos de los Censos Económicos, CE (2019), “... había 6,373,169 establecimientos, con 36,038,272 empleos generados". En lo relativo a Veracruz, se tiene registro del Directorio Estadístico Nacional de Unidades Económicas, DENUE (2018), que "las microempresas son 284,862 que corresponden al $94.50 \%$ de los establecimientos, las pequeñas 13,616 que son el $4.52 \%$, las medianas son 2,337 que corresponden el $0.78 \%$ ”. En la ciudad de Xalapa, de acuerdo con el Directorio Empresarial lista de empresas: "en Xalapa, Veracruz 26, I 36 registros entre MiPymes, Pymes y empresas en general".

Del mismo modo, los Censos Económicos (CE) (2019) indican que "el 37.2\% del personal ocupado laboró en micronegocios ( 0 a 10 personas), $30.7 \%$ en Pymes (II a 250 personas) y $32.1 \%$ en unidades económicas grandes (más de 250 personas)”, permitiéndonos estos datos apreciar la conformación estratificada por tamaño de empresa. Lo antes señalado es de manera general, en el caso particular a este proyecto, el número de trabajadores de acuerdo con el sector económico comercial a estudiar se establece de acuerdo con lo siguiente: para micros hasta 10 personas, en Pymes desde II hasta 100 personas y en unidades económicas grandes a partir de 100 personas.

Pese a lo relevante de los datos anteriores, el nivel de competitividad que muestran las MiPymes del sector económico comercial resulta demasiado bajo. Por lo cual, es necesario señalar la relevancia de la incorporación de $\mathrm{Tl}$ en las actividades prioritarias en las grandes empresas, quienes enarbolan su éxito en actividades de investigación, desarrollo e innovación tecnológica, dirigiéndose en la mayoría de los casos, al éxito empresarial y los beneficios inmanentes a este. Por el contrario, la falta de implementación de $\mathrm{TI}$ en las actividades clave de las MiPymes comerciales evitan potenciar su competitividad interna y hacia el mercado en que se insertan.

Derivado de esta información, el punto relevante para el estudio realizado en el presente proyecto de investigación es conocer la relación 
existente entre el nivel de integración de TI y la competitividad con que se desempeñan las MiPymes del sector económico comercial, en la ciudad de Xalapa, Veracruz, México. De este análisis, se espera poder apreciar el impacto que tiene la integración de $\mathrm{Tl}$ en las actividades claves de las MiPymes del señalado sector económico, dentro de sus procesos y procedimientos establecidos. Se permitirá vincular los datos obtenidos en las fuentes de información acerca de las dimensiones que permitan aclarar el escenario tecnológico que presenten las MiPymes en los diferentes momentos de la intervención durante la temporalidad del proyecto.

De esta manera, el objetivo de la presente será, una vez realizado el respectivo diagnóstico empresarial, proponer un método alternativo estratégico de $\mathrm{TI}$ en MiPymes comerciales en Xalapa, Ver., México, con base en sus necesidades específicas, esto, para incrementar su competitividad empresarial.

Para efectos de la investigación llevada a cabo, el método utilizado es de tipo deductivo, pues se analiza la situación del contexto nacional y se dirige hacia el enfoque local, de la ciudad de Xalapa, Ver., y dentro del sector económico comercial propio de las MiPymes abordadas.

\section{DESARROLLO}

\section{Marco teórico y estado de la literatura}

Para conceptualizar la Teoría general de sistemas (TGS), Bertalanffy (Arnold, 1998) “reconoce que la teoría de sistemas comprende un conjunto de enfoques que difieren en estilo y propósito, entre las cuales se encuentra la teoría de conjuntos (Mesarovic), teoría de las redes (Rapoport), cibernética (Wiener), teoría de la información (Shannon y Weaver), teoría de los autómatas (Turing), teoría de los juegos (Von
Neumann), entre otras". De igual manera él distingue en la filosofía de sistemas una ontología de sistemas, una epistemología de sistemas y una filosofía de valores de sistemas.

El estudio de los sistemas desde la perspectiva organizacional establece que un sistema es un conjunto de elementos interrelacionados. El mínimo de elementos es de dos y cada uno de los elementos del sistema se conecta con cada uno de los otros elementos directa o indirectamente (Ackoff, 197I). Se puede definir como el conjunto de datos que, transformados o modificados, tienen un valor para aquellos usuarios que hacen uso de ellos. Dicho de otra manera, y con base en una perspectiva de sistemas para Johannsen, (Ros, 1993), "la cantidad de información que permanece en el sistema (...) es igual a la información que existe más la que entra, es decir, hay una agregación neta en la entrada y la salida no elimina la información del sistema" (p. 78). La información es la más importante corriente neguentropía de que disponen los sistemas complejos.

A su vez, Ros (1993, p. 4), define un sistema de información "como una colección de personas, procedimientos y equipos diseñados, construidos, operados y mantenidos para recoger, registrar, procesar, almacenar, recuperar y visualizar información”. Otra conceptualización, de Andreu, Ricart y Valor (199I) es conjunto formal de procesos que, operando sobre una colección de datos estructurada de acuerdo a las necesidades de la empresa, recopila, elabora y distribuyen selectivamente la información necesaria para la operación de dicha empresa y para las actividades de dirección y control correspondientes, apoyando, al menos en parte, los procesos de toma de decisiones necesarios para desempeñar funciones de negocio de la empresa de acuerdo con su estrategia. Es 
preciso señalar que los sistemas de información se agrupan según su utilidad en los diferentes niveles de la organización empresarial. Esta organización consta de 4 niveles básicos de acuerdo con K y J Laudon (1996): un nivel operativo referido a las operaciones diarias de la organización, un nivel del conocimiento que afecta a los empleados encargados del manejo de la información (generalmente el departamento de informática), un nivel administrativo (abarcaría a los gerentes intermedios de la organización) y un nivel estratégico (la alta dirección de la empresa)", de esta manera se pretende alcanzar resultados en la ejecución. El sistema de información establece cuáles son las necesidades de información de las empresas, cómo las va a solucionar y qué medios (tecnologías de información) va a emplear. Se hace oportuno pues considerar un proceso para determinar favorablemente la selección del sistema que requiere la empresa en cuestión con los atributos necesarios.

Tecnología de Información se refiere a la tecnología basada en computadora para el almacenamiento, acceso, procesamiento y comunicación de información (Molloy, 1995, PP 283-3II). En otras palabras, los equipos informáticos, software y equipos de telecomunicaciones constituyen las tecnologías de información.

Es importante especificar acerca de la tecnología, para Arner et al (2015) la tecnología análoga, la cual se refiere a la traducción de datos para su transmisión en forma de impulsos eléctricos de amplitud variada; mientras que la tecnología digital traduce la información a través de un sistema binario $(0$ y l $)$ donde cada bit es representativo de dos distintas amplitudes. Un ejemplo de señal análoga es la voz humana mediante un micrófono y un ejemplo de señal digital se encuentra en las computadoras personales.
Hoy día, se habla ya de la informática cuántica (QC) utiliza qubits, como lo menciona Shu, (2014) los cuales permiten que una computadora vaya más allá de simplemente dos estados para almacenar una cantidad enorme de información mientras que utiliza menos energía. Las computadoras cuánticas no reemplazarán a las computadoras tradicionales, sino que podrán resolver problemas computacionalmente difíciles. Además de encontrar respuestas rápidas y alcanzar la precisión de la inteligencia artificial. Incluso, estas computadoras podrían hacer que la tecnología parezca intuitiva. Ejemplificando, la tecnología para detectar fraudes o lavado de dinero es un área muy adecuada para la informática cuántica. No importa lo rápidas y poderosas que pueda llegar a ser las computadoras digitales, pues persisten algunos problemas, como en el caso de las finanzas que no se pueden resolver.

En este escenario, se vislumbra un ejercicio responsable y ético de las tecnologías de la información en búsqueda de dar solución a la diversidad de problemáticas en las diversas áreas de la empresa, en una vertiente progresista.

Se define como el proceso de manejar todas aquellas actividades que habiliten a la empresa para hacer el uso más eficiente de la tecnología generada internamente y de la adquirida a terceros, así como de incorporarla a los nuevos productos (innovación de producto) y a las formas en que los producen y se entregan al mercado (innovación de proceso). Se encarga de los problemas de decisión a todos los niveles relacionados con la creación y utilización de activos y capacidades tecnológicas; sus impactos sobre los individuos, organizaciones, sociedades y naturaleza; y la conciliación de las consecuencias económicas, sociales y ambientales de las innovaciones tecnológicas (Bayraktar, 
1990). En términos comerciales, lo enuncia Arévalo (2007), la información es objeto de mercadeo, se compra y se vende, y puede proveer a aquellos que la tienen unos beneficios reales y efectivos. Esta prueba es tal que los dirigentes de las primordiales organizaciones han diseñado políticas para gestionar este recurso en beneficio de los intereses de su entidad. No obstante, a veces además confundidos frente a este caso, simplifican la administración de la información con la sencilla unión de tecnologías de la información de última generación, que si bien poseen una trascendencia importante como herramientas para la administración de la información en sí misma solamente tienen la posibilidad de considerarse un soporte para ofrecer cobertura a la administración de la información.

Queda abierto el análisis de dicha información con el fin de utilizarla para métricas y a su vez, para toma de decisiones empresariales tras su interpretación, derivado como se mencionó anteriormente, de un diagnóstico oportuno.

Al respecto, Lundvall (Contreras, 2019), menciona que los procesos de innovación no solo incluyen las nuevas tecnologías, sino que también comprenden las innovaciones de productos y de procesos, así como otras formas de innovación no tecnológicas, como las desarrolladas en las organizaciones de servicios.

En lo concerniente a la gestión empresarial basada en la creatividad, la gestión tecnológica y la innovación debería estar presente en las empresas modernas de cualquier sector de la economía, más que nada en pequeñas y medianas organizaciones que tienen que insertarse a un mundo competitivo exigente. Esta administración camina de la mano con la tecnología de información y comunicación, posibilita navegar satisfactoriamente en el mercado empresarial $y$, de modo que, coopera al desarrollo económico y social del ambiente en que se desenvuelve. Las organizaciones como unidades de producción crean puestos de trabajo, impulsan y proporcionan forma a la innovación, aceleran los cambios estructurales, aportan a la economía del territorio. (Quispe, 2017).

En la misma vertiente, en la mayor parte de las organizaciones, es preciso remplazar la infraestructura tecnológica, para la implementación de nuevos sistemas automatizados que posibiliten la concentración de información que posibilite la toma de elecciones oportunas que coadyuven al incremento empresarial. Es fundamental señalar que el nivel de capacitación de los relacionados en la generación de información ayuda al buen desenvolvimiento del sistema. (Quispe, 20I7).

En el marco empresarial, los esfuerzos por mejorar la competitividad se ha permitido una mirada hacia las tecnologías de la información en busca de alinear las estrategias con los procesos clave de la empresa o negocio. Para ello, se evidencia el aumento importante de adquisiciones de paquetes de programa empresariales como por ejemplo el ERP (Enterprise Resource Planning), con el que los directivos de las organizaciones esperan tener integradas cada una de las zonas, secciones o apartamentos de la organización que secundan la venta de sus productos. Un ERP es un sistema informático de gestión gerencial que posibilita evaluar, mantener el control de y gestionar de forma más simple y rigurosa una organización. (Quispe, 2017).

Describe Reyes, Dora, et al (2016), “por lo general, como en cualquier sistema informático, se puede llegar optar por dos opciones. La primera es un software Empresarial de Planeación de Recursos (ERP) 
a la medida; la segunda, una solución genérica o estándar" (p. 5I).

Las consideraciones a este punto son realizadas por la capacidad de inversión en equipo, así como en la capacidad de conocimiento para su óptimo aprovechamiento y el tamaño de la empresa.

\section{Marco conceptual}

Para abordar el enfoque de la organización, se considera a Taylor y su obra más conocida, Principios de la administración científica, en la que sugirió un sistema de trabajo basado en cuatro principios:

I. Desarrollo de una ciencia de medición del trabajo de las personas, que reemplazaba a las viejas prácticas empíricas.

2. Un proceso de selección científica, entrenamiento y desarrollo de los trabajadores, que sustituyera a los antiguos esquemas con los cuales los trabajadores se entrenaban por sí mismos lo mejor que podían.

3. Un esfuerzo cooperativo de los trabajadores para asegurar que todo el trabajo se realizara conforme a los principios de la administración científica.

4. La idea de que el trabajo y la responsabilidad son compartidos tanto por la administración como por el trabajador" (2009).

Con ello, Taylor representó el parteaguas de la administración del siglo XX. Más tarde en Francia, Henry Fayol fundamentó la concepción de que toda organización estaba basada en cinco funciones básicas: seguridad, producción, contabilidad, comercialización y administración, además de catorce principios que deberían ser observados para operar con eficiencia.
Posteriormente, las teorías de la organización han pasado por estadios que permiten apreciar las adaptaciones y $\circ$ modificaciones a los pensamientos contemporáneos de cada época, llegando a nuestros días con teorías como la de sistemas la cual ya ha sido mencionada en el presente documento.

Este transcurrir, nos lleva a teorías más actuales como la teoría de la población ecológica (Hannan y Freeman, 1977, 1984), dada en dos tiempos. En su primer trabajo se enfocaron en la población y en el segundo en la empresa, dando énfasis a la importancia del entorno en la subsistencia (Rivas, 2009). En prospectiva, se llega a enumerar la Teoría de los sistemas complejos adaptativos de S. Kauffman (1993, 1995), señalando que la complejidad es el resultado de procesos autoorganizativos, cuya base, a su vez, son procesos autocatalíticos. Un sistema de autorregulación y con su entorno. (Rivas, 2009).

Desde la perspectiva de procesos, para Larrea (Herrera, 2018) indica que el proceso de calidad en las empresas requiere de elementos: querer (motivación y educación de actitudes), poder (implantar procesos de formación que cualifiquen técnicamente) y saber (desarrollar sistemas de información) (Blázquez, 20I3).

Para destacar en la esfera empresarial, se debe mejorar la competitividad, en un sentido global se refiere a la competitividad internacional, derivándose en escala nacional entre los estados y a su vez en el ámbito local entre las empresas de la ciudad correspondiente. Señala Ibarra (2017) "El nivel de competitividad internacional de un país se relaciona estrechamente con empresas altamente productivas que aprovechan las ventajas competitivas que genera el país por medio de sus instituciones, políticas, infraestructura y cultura" (p. III). 
Por otro lado, Porter (199I) sostiene que la competitividad de una nación se debe a que sus empresas son altamente productivas gracias al uso eficiente de sus recursos humanos, naturales y de capital. Para lo cual diseñó el modelo "diamante de competitividad" que permite visualizar las ventajas competitivas de un país en relación con otros; aunque también se puede llevar a cabo a escala industria o empresa, por medio del análisis de las condiciones de los factores, de la demanda, estrategia, estructura y rivalidad empresarial, así como las industrias relacionadas y de apoyo. La conjugación de estos factores da como resultado el nivel de competitividad que se presenta en una economía (Chacón, 2007).

Mientras tanto, Saavedra y Milla (20I2) comentan que el término competitividad no posee una definición específica. Existe una falta de consenso para definir[la] conceptualmente, debido a la amplitud de su significado, que puede abarcar desde el nivel de la empresa, sector, nación y ámbito supranacional; así como, por la naturaleza cualitativa y cuantitativa de sus factores carece de límites precisos en el nivel de análisis y en las diversas metodologías de medición" (Chacón, 2007).

En este sentido, la presente investigación se enfocará en una delimitación micro, respecto del sector económico empresarial en la ciudad de Xalapa, Ver., de donde se considerará la población y por añadidura, la muestra seleccionada.

Considerando las empresas existentes en el mercado en pugna, la competitividad empresarial se deriva de la ventaja competitiva que tiene una empresa a través de sus métodos de producción y de organización (reflejados en precio y en calidad del producto final) en relación con los de sus rivales en un mercado específico (Chacón, 2007).

Las empresas compiten para ganarse la preferencia de los consumidores, proveedores $y$ clientes. En tal sentido, para ser competitivas, necesitan tener un desempeño mayor que otras, pues se disputan los mismos clientes. Una organización es competitiva cuando tiene alguna ventaja sobre sus competidores. Con base en este enfoque (Saavedra, 2017) las empresas se convertirán en competitivas a través de dos requisitos: I) encontrarse en un entorno de presión de competencia que las obligue a mantener esfuerzos sostenidos para mejorar sus productos y su eficiencia productiva $y, 2$ ) insertarse en redes articuladas apoyadas por servicios eficientes del gobierno $y$ de las instituciones".

En cuanto a la competitividad, Gómez (1994) afirma que "sin mayores refinamientos conceptuales se entiende por competitividad como la capacidad de producir más a menor costo o con mayor calidad que los demás" (p. 12). Consecuentemente, en el aspecto macro, el Foro Económico Mundial (2016) que ha medido la competitividad entre países desde 1979 la define como el conjunto de instituciones, políticas y factores que determinan el nivel de productividad de un país.

En el contexto nacional, para Morales (20II):

el concepto de competitividad se refiere a las empresas que exportan productos y compiten en el mercado internacional. En el mercado interno, una empresa es competitiva cuando esta cuenta con herramientas que les ofrecen una ventaja frente a sus competidores (Pp. 49$50)$. 
Para Ramírez (2017) los factores que más impactan en la competitividad actualmente son:

el recurso humano, la innovación, la tecnología en los procesos, la administración estratégica, la calidad, las redes sociales y la seguridad o estado de derecho. Otros factores que hay que considerar en un futuro cercano son: La responsabilidad social, logrando la certificación de empresa socialmente responsable (ESR). El costo país quiere decir, que entre mejor infraestructura tenga una nación, más económica es realizar operaciones comerciales, y finalmente el de las incubadoras de negocio, se apoya a los emprendedores mediante consultoría calificada para que inicien operaciones y aumenten sus posibilidades de éxito (p. I).

Visto como empresa, el concepto que maneja Rubio (2006) es la función de una organización para, rivalizando con otras, conseguir una postura competitiva conveniente que posibilite la obtención de un funcionamiento mayor a las organizaciones de la competencia.

Para conceptualizar este término, la OCDE (2005) establece que

la innovación es la introducción de un nuevo o significativamente mejorado producto (bien o servicio), de un proceso, de un método de comercialización o de un nuevo método organizativo, en las prácticas internas de la empresa, la organización del lugar del trabajo o las relaciones exteriores (p. 56).
Con ello nos establece una constante dentro del ámbito de las organizaciones respecto de sus procesos cotidianos.

Por su parte, Dessler (2009) explica que una ventaja competitiva se define como todos los factores que permiten que una organización distinga su producto o servicio de los de la competencia, con el objetivo de aumentar su participación en el mercado.

A este respecto, Porter (1985) afirma que las consecuencias de la ventaja competitiva son la obtención de rendimientos superiores.

\section{MÉTODO}

El tipo de investigación es de tipo exploratoria descriptiva y cuantitativa. Se inició con una investigación de tipo teórica, de revisión de la literatura académica con énfasis en artículos científicos para continuar con la aplicación del instrumento y finalizar con una síntesis sobre resultados.

El presente proyecto se dirige a las MiPymes del sector económico comercial, que realizan actividades en busca de la optimización de los -generalmente escasosrecursos que dispone, para estimular su productividad y por consecuencia, incrementar su competitividad empresarial. En esta búsqueda de mayor competencia, se considera la integración de $\mathrm{TI}$ en sus aspectos esenciales, por tanto, se espera lograr como objetivo general: Proponer alternativas estratégicas de $\mathrm{TI}$ en las actividades claves de las MiPymes comerciales en Xalapa, Ver., México, de acuerdo con sus características y necesidades requeridas, para incrementar su competitividad empresarial. 
En cuanto a los objetivos específicos, son cuatro:

- Conocer la infraestructura en tecnologías de la información con que operan las MiPymes del sector económico comercial de la ciudad de Xalapa, Ver., México, para comprender sus procesos claves.

- Identificar la integración de sistemas de información y herramientas digitales adecuados a las actividades cotidianas $y$ a las necesidades de administración de recursos de las MiPymes señaladas.

- Aumentar la generación de valor al implementar TI en las MiPymes señaladas, en sus diferentes áreas y actividades claves.

- Mejorar la competitividad empresarial a través de la implementación de herramientas digitales óptimas para la gestión de MiPymes del sector económico comercial en la ciudad de Xalapa, Ver., México, para el éxito empresarial.

Como parte de planteamiento del problema en la ciudad de Xalapa, Veracruz México, existe un número considerable de MiPymes dentro del sector económico comercial, el cual se caracteriza por ser de los más activos en la economía a nivel nacional, con suma importancia para los factores de empleabilidad y de aporte al PIB, como ya se ha venido señalando. Derivado de la importancia que tiene este sector comercial y del número de establecimientos existentes que abarrotan la imagen citadina en sus principales calles $y$ avenidas, como en las diferentes plazas comerciales y zonas de mercados a lo largo y ancho de la capital veracruzana, no obstante, se logra apreciar, a simple vista y a nivel de consumidor, algunas debilidades y amenazas que enfrentan en su día a día de acuerdo con grandes áreas de oportunidad de acuerdo al giro tan diverso que se puede encontrar en este abanico empresarial.

Partiendo de lo anterior se puede establecer que el nivel de competitividad de las MiPymes del sector económico comercial en la ciudad de Xalapa, Ver., México, resulta bajo, esto derivado de factores como la falta de implementación de Tecnologías de Información (TI) en sus actividades y procesos claves, lo cual, limita el incremento de la competitividad empresarial en el mercado en el que realizan su actividad comercial.

Ante ello, se hace presente la oportunidad de realizar un estudio que permita esclarecer la situación anterior, para poder así determinar ciertas recomendaciones puntuales y estratégicas que generen un impacto positivo en la competitividad de las MiPymes señaladas.

Se consultó el estudio de MiPymes nacionales, de acuerdo con la información acerca de la estratificación del Instituto Nacional de Estadística, Geografía e Informática 2019 (INEGI 2019), así como, del Sistema de Clasificación Industrial de América del Norte, México 2018 (INEGI, 2018), para lo correspondiente a la asignación del sector económico.

Se llevó a cabo la observación, de acuerdo con el Directorio Empresarial de la lista de empresas en Xalapa, Veracruz, a las MiPymes, Pymes y empresas en general. Más específicamente, una población de 26, I36 entre MiPymes, Pymes y empresas en general, no se lograron encontrar fuentes secundarias de información que permitieran identificar la población MiPymes del sector económico comercial, de la ciudad de Xalapa, Ver., México. Por lo tanto, se eligió optar por la realización del cálculo de muestreo para una población infinita, tal como se muestra en la Tabla I. 
Tabla I

Datos para el cálculo de la muestra

\begin{tabular}{cc}
\hline Parámetro & Insertar Valor \\
\hline Z & 1.645 \\
$P$ & $50 \%$ \\
$Q$ & $50 \%$ \\
e & $8 \%$ \\
\hline
\end{tabular}

Fórmula:

$$
n=\frac{Z^{2} * p * q}{e^{2}}
$$

En donde:

$\mathrm{n}=$ Tamaño de muestra buscado

$Z$ = Parámetro estadístico que depende de $\mathrm{N}$ (grado de precisión)

e $=$ Error de estimación máximo aceptado

$\mathrm{P}=$ Probabilidad de que ocurra el evento

$q(I-p)=$ Probabilidad de que no ocurra el evento

Tamaño de la muestra de 106 MiPymes comerciales de la región de Xalapa, Veracruz. Se empleó una encuesta estructurada de 35 preguntas, en las que se plantearon dimensiones en cuanto a la planeación estratégica empresarial, integración de TI, Productividad, Marketing y, por último, la Relación con el cliente y proveedores.

Esta encuesta se realizó a propietarios, gerentes o encargados del establecimiento en turno, esto de manera aleatoria, procurando una diversidad de giros de este sector económico comercial. La escala de Likert implementada para este efecto contenía los valores: Nunca, Raramente, Ocasionalmente, Frecuentemente y Muy Frecuentemente, mismos a los que les correspondía un valor numérico de $\mathrm{I}$ a 5 , según corresponda.
Este instrumento fue validado con el alfa de Cronbach para determinar la fiabilidad de este a partir de treinta y cinco encuestas, mismas que se consideran como parte de la muestra. Tras la aplicación del instrumento para el cálculo de alfa de Cronbach, se obtiene que la fiabilidad de este es de 0.947 , lo cual, de acuerdo a la escala, nos da un resultado confiable de dicho instrumento aplicado a la muestra en turno (véanse Tabla 2).

\section{Tabla 2}

Análisis de fiabilidad que arrojó el alfa de Cronbach

\begin{tabular}{ccc}
\hline $\begin{array}{c}\text { Alfa de } \\
\text { Cronbach }\end{array}$ & $\begin{array}{c}\text { Alfa de Cronbach } \\
\text { basada } \\
\text { en elementos } \\
\text { estandarizados }\end{array}$ & $\begin{array}{c}\text { N de } \\
\text { elementos }\end{array}$ \\
\hline .947 & .949 & 35 \\
\hline
\end{tabular}

\section{RESULTADOS}

En el presente apartado se mostrarán los resultados descriptivos más significativos de la investigación. Se inicia con la Tabla 3 en la que se pueden visualizar las estadísticas generales del elemento, para este caso, el resultado de todos los ítems del instrumento que se aplicó.

\section{Tabla 3}

Estadísticas de elemento de resumen

\begin{tabular}{lccccccc}
\hline & Media & Mínimo & Máximo Rango / Mínimo & Varianza & $\begin{array}{c}\text { N de } \\
\text { elementos }\end{array}$ \\
\hline $\begin{array}{l}\text { Medias de } \\
\text { elemento }\end{array}$ & 3.105 & 2.260 & 3.885 & 1.625 & 1.719 & .157 & 33 \\
$\begin{array}{l}\text { Varianzas } \\
\text { de }\end{array}$ & 2.119 & 1.163 & 3.417 & 2.254 & 2.938 & .213 & 33 \\
elemento & & & & & & & \\
$\begin{array}{l}\text { Covarianz } \\
\text { as entre } \\
\text { elementos }\end{array}$ & .726 & -.012 & 2.636 & 2.647 & -222.556 & .086 & 33 \\
$\begin{array}{l}\text { Correlacio } \\
\text { nes entre } \\
\text { elementos }\end{array}$ & .347 & -.007 & .813 & .821 & -108.687 & .017 & 33 \\
\hline
\end{tabular}


A continuación, se muestran los resultados descriptivos referente a la dimensión tecnológica del instrumento aplicado.

Como se visualiza en la Figura I, de los encuestados, $36.46 \%$ mencionó que su empresa no hace uso de equipo de cómputo y de sistemas de administración empresariales, por el contrario, un $35.42 \%$ mencionó que sí emplean ambos elementos tecnológicos señalados, lo que lleva a interpretar la necesidad de implementar $\mathrm{TI}$ para mejorar la competitividad de las MiPymes.

\section{Figura I}

Utiliza equipo de cómputo con un ERP integrado

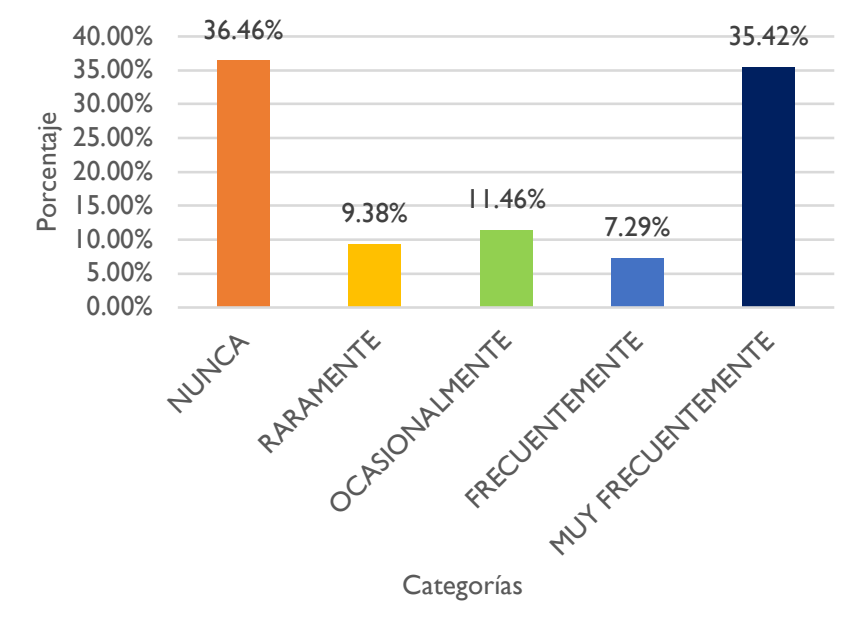

Uno de los cuestionamientos fue si las MiPymes, además de utilizar equipo de cómputo con sistema de administración empresarial integrado, cuenta con accesorios (periféricos) como: caja para cobro, lector de código de barras, impresora de tickets de compra, entre otros. En la Figura 2, se aprecia que $44.79 \%$ de los establecimientos encuestados no dispone de accesorios para su sistema de administración empresarial, mientras que $36.46 \%$ sí cuenta con tales elementos, por lo que se interpreta la necesidad para implementar estos periféricos tecnológicos, en beneficio de su

\section{Figura 2}

Utiliza periféricos complementarios al ERP

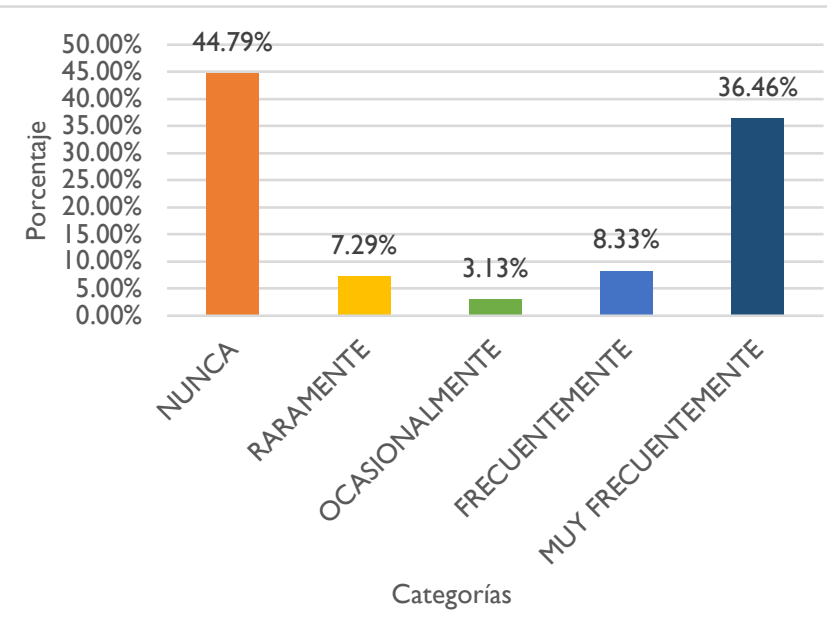

Se logra interpretar en la Figura 3, que 51.04\% de los establecimientos utilizan las terminales de cobro para tarjetas bancarias en sus transacciones de compra venta, así como un $18.75 \%$ lo utilizan frecuentemente, por el contrario, un $19.79 \%$ no se valen de este medio digital para realizar el cobro de sus transacciones. De acuerdo con el tipo de operaciones que se realizan con estas herramientas tecnológicas para el cierre de las transacciones comerciales, se puede observar la necesidad para implementarlas en beneficio de la competitividad de los establecimientos.

\section{Figura 3}

Cuentan con terminal para cobro con tarjetas bancarias

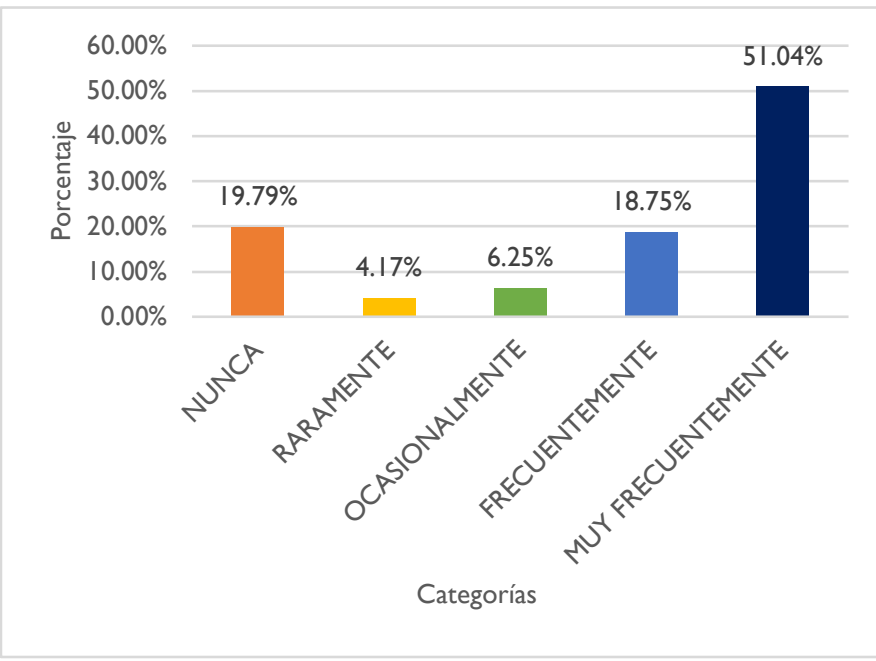
competitividad. 
En la Figura 4 se observa un $37.50 \%$ de personal capacitado en el manejo de los equipos tecnológicos con los que cuentan las MiPymes, seguido de un $23.96 \%$ que respondió contar con una capacitación frecuente, mientras que el $18.75 \%$ mencionó no contar con la capacitación adecuada para el manejo de dichos equipos, este último porcentaje, aunque menor, muestra la necesidad de la capacitación respecto de la TI con que operan las MiPymes, en beneficio de su competitividad.

\section{Figura 4}

El personal que opera TI cuenta con capacitación adecuada

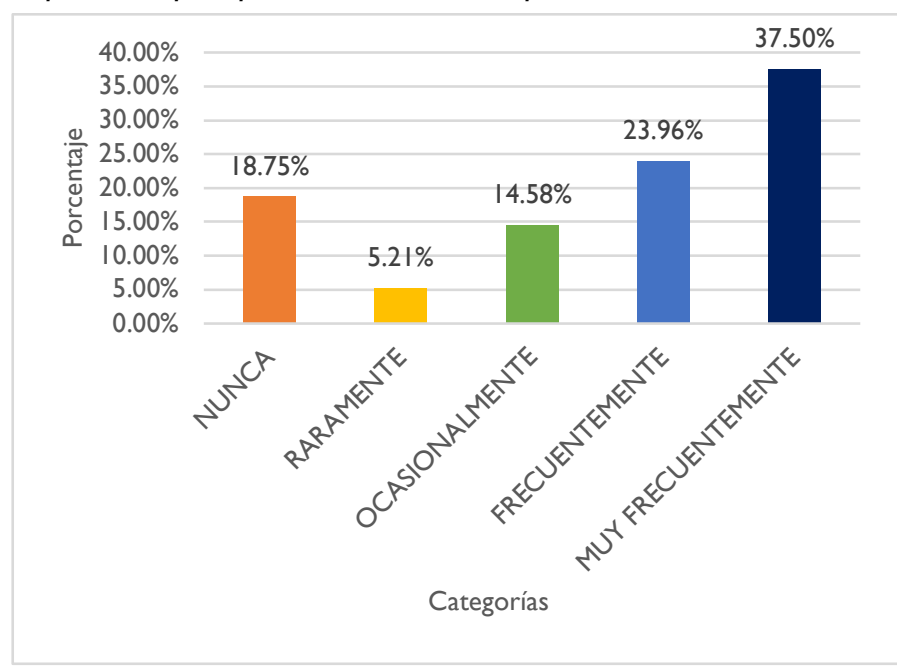

De las MiPymes encuestadas $42.71 \%$ emplea otras herramientas digitales a las incluidas en el ERP para llevar a cabo el inventario y control de productos que vende o servicios que realiza. Por el contrario, $20.83 \%$ nunca las ha utilizado y $17.71 \%$ rara vez las ha utilizado, por lo que se observa la necesidad de incluir este tipo de herramientas (véase Figura 5).

En la Figura 6 se observa la falta de uso cotidiano de una página web empresarial en las MiPymes, en un $28.13 \%$, en contraparte, el $23.96 \%$ de estas no cuentan con ese sitio web en su accionar diario. Por tanto, se muestra la necesidad de contar con una página web de uso cotidiano en concordancia con la competitividad de estas.

\section{Figura 5}

Utilizan otras herramientas digitales a las incluidas en el ERP

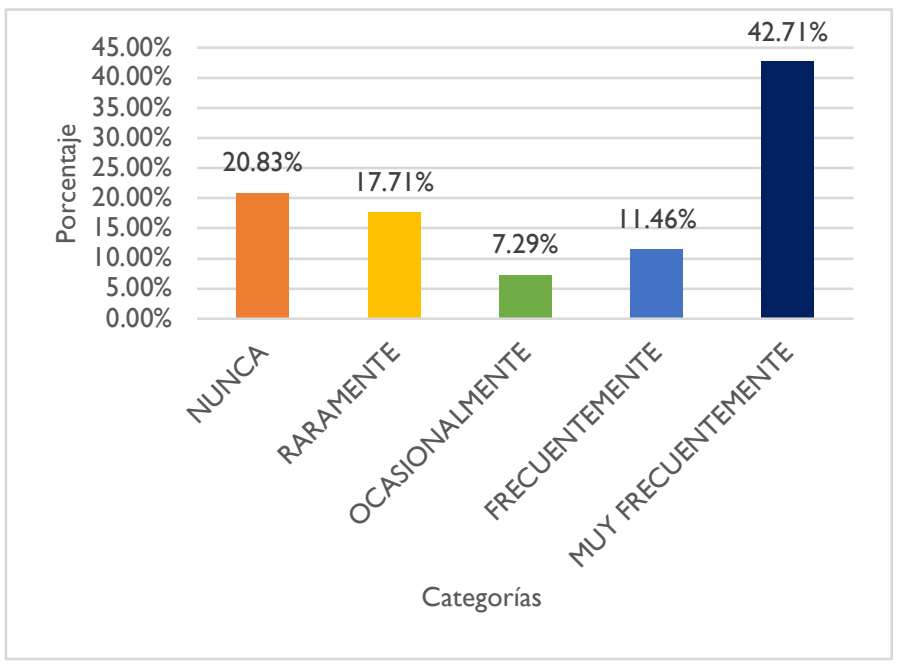

\section{Figura 6}

Utilizan cotidianamente una página web empresarial

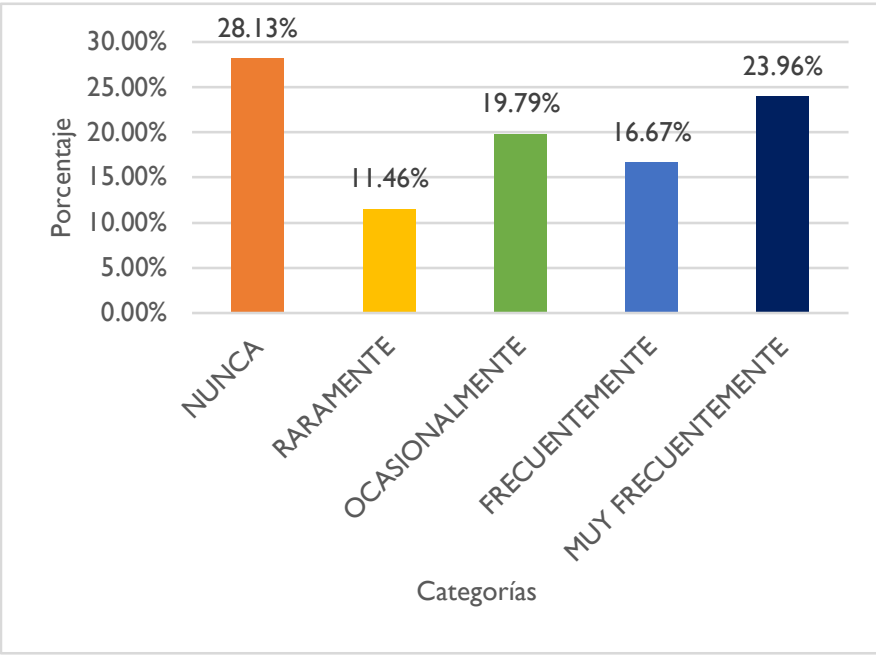

Se aprecia en la Figura 7, un $38.54 \%$ de las MiPymes encuestadas que se apoyan en las redes sociales para darse promoción, mientras el $16.67 \%$ nunca las ha utilizado para tal efecto. Esto muestra una oportunidad para integrar este apoyo digital para incremento de la competitividad en los establecimientos señalados. 


\section{Figura 7}

Utilizan redes sociales para su promoción

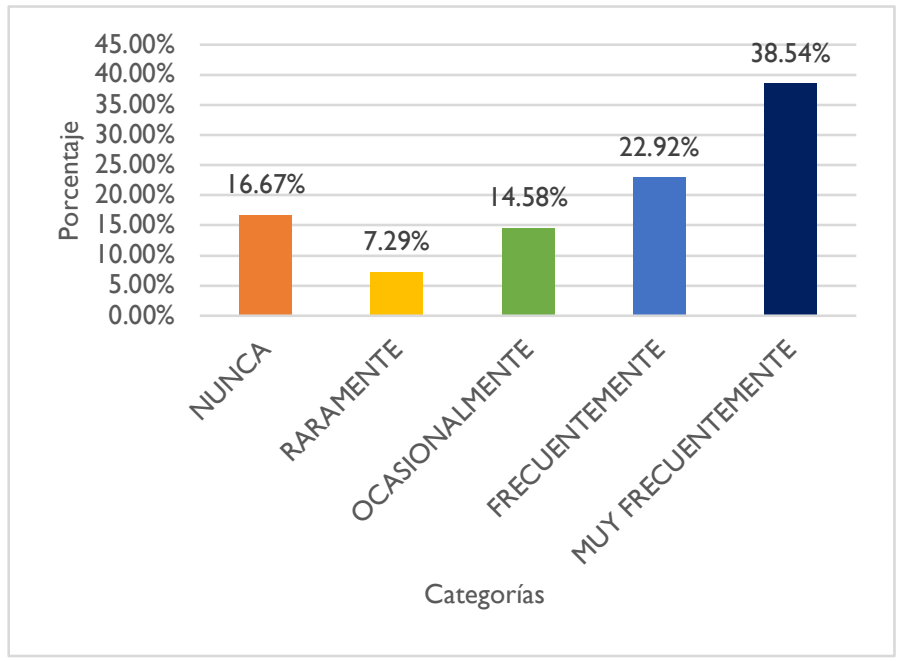

La Figura 8 muestra que un 42.71\% de MiPymes no emplean personal para la gestión de redes sociales y un $22.92 \%$ que sí lo hacen. Esto muestra la necesidad de contar con personal que gestione las redes sociales en beneficio de la competitividad.

\section{Figura 8}

Emplean una empresa o persona en gestión de redes sociales

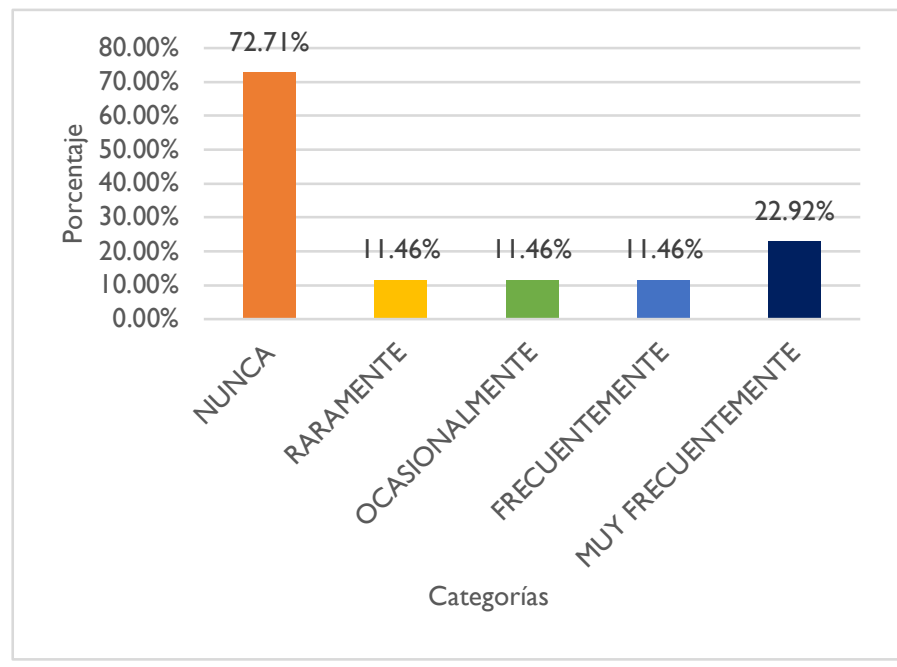

Finalmente se visualiza en la Figura 9, que un $52.08 \%$ no cuentan con comercio electrónico para realizar ventas online, por otra parte, el $18.75 \%$ muy frecuentemente cuentan con este tipo de comercio. De esta manera se considera necesario para impulsar la competitividad del establecimiento integrar esta actividad digital.

\section{Figura 9}

Cuentan con una tienda online (comercio electrónico)

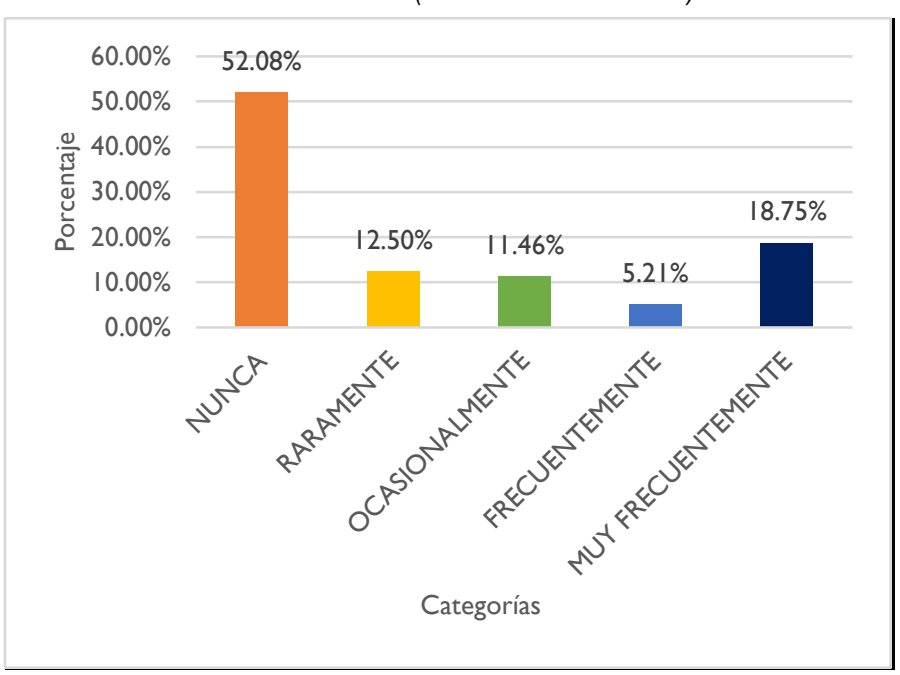

\section{ANÁLISIS CORRELACIONALES DE DATOS}

Además de los resultados descriptivos de la investigación, se llevó a cabo un análisis correlacional de datos por medio de la estadística inferencial no paramétrica, empleando la metodología de Tau-b de Kendall para tablas cuadradas El análisis se hizo con el uso de dos variables una de la dimensión planeación estratégica empresarial y otra de la dimensión integración de TI, con el objetivo de determinar si existe una relación entre la Planeación Estratégica y la implementación de TI que mejore la competitividad.

Se analizó si ¿existe alguna asociación entre llevar a cabo la Planeación Estratégica con la implementación de un sistema ERP para mejorar la competitividad de las empresas? Las hipótesis que se formularon son:

- H0: No Existe relación significativa entre llevar a cabo una Planeación Estratégica y la implementación de un Sistema ERP que mejore la competitividad de las empresas. 
- HI: Existe relación significativa entre llevar a cabo una Planeación Estratégica y la implementación de un Sistema ERP que mejore la competitividad de las empresas.

- Si la sig. p-valor es $<0.05$ se rechaza $\mathrm{H} 0$; en caso contrario se acepta.

- Método Tau-b de Kendall

\section{Tabla 3}

Correlación Planeación Estratégica - Implementación ERP

\begin{tabular}{|c|c|c|c|c|}
\hline \multicolumn{5}{|c|}{ Correlaciones } \\
\hline \multirow{7}{*}{$\begin{array}{c}\text { Tau_b } \\
\text { de } \\
\text { Kendall }\end{array}$} & \multirow{4}{*}{ PE } & & PE & ERP \\
\hline & & $\begin{array}{l}\text { Coeficiente de } \\
\text { correlación }\end{array}$ & 1.000 & .359 \\
\hline & & Sig. (bilateral) & $\cdot$ & .001 \\
\hline & & $\mathrm{N}$ & 106 & 106 \\
\hline & \multirow{3}{*}{ ERP } & $\begin{array}{l}\text { Coeficiente de } \\
\text { correlación }\end{array}$ & .359 & 1.000 \\
\hline & & Sig. (bilateral) & .001 & . \\
\hline & & $\mathrm{N}$ & 106 & 106 \\
\hline
\end{tabular}

Según el p-valor "Sig. (Bilateral)" =.00I <.05, lo que conlleva a rechazar la $\mathrm{HO}$ concluyendo que hay una asociación altamente significativa entre las variables de Planeación Estratégica que operan en las MiPymes y la implementación de los sistemas ERP, es decir, los que realizan la planeación estratégica enfocado a la implementación de sistemas ERP mejoran su competitividad. Esto queda evidenciado con el valor de coeficiente de correlación $t=.0 .359$, el cual se interpreta como una correlación positiva moderada entre las variables.

Con los datos anteriores podemos mencionar, que existe una asociación entre las variables, sin embargo, la correlación es moderada, por lo que no podemos ser concluyente en la afirmación de la correlación existente, recordando que la correlación de Kendall son pruebas de hipótesis y medida de correlación a través de los índices

\section{PROPUESTA METODOLÓGICA ESTRATÉGICA DE IMPLEMENTACIÓN DE TI}

Con base en el análisis realizado al instrumento aplicado en las MiPymes del sector económico comercial de la ciudad de Xalapa, Ver., México, y en apego a lo ítems seleccionadas para su interpretación, se logra apreciar una oportunidad para integrar $\mathrm{TI}$ en diferentes áreas y actividades clave de los establecimientos señalados, en busca de generar un impacto positivo directo a su competitividad, ya que existen porcentajes que así lo determinan.

Por lo antes señalado, se fundamenta realizar una propuesta metodológica para integrar alternativas de $\mathrm{TI}$ de acuerdo con la oportunidad detectada, esto en áreas consideradas clave para el funcionamiento cotidiano de las actividades que se desarrollan en las MiPymes señaladas, la propuesta metodológica se enuncia a continuación:

I. En principio, se buscará conocer la infraestructura en TI disponible en la MIPYME comercial y el nivel de conocimiento acerca de esta para su manejo cotidiano, así se tendrá un diagnóstico con base en las características y las necesidades específicas del establecimiento, el cual será el punto de partida.

2. Posterior a ello, se considera como alternativa de solución integrar en el equipo de cómputo un sistema de administración de recursos empresariales (ERP). Este sistema a medida se considera así con base en la estratificación tanto de micros, pequeñas como de medianas empresas, pudiendo optar por un ERP de uso libre o genérico con la posibilidad de ser escalable en proporción al crecimiento del establecimiento, así como de los requerimientos operativos de los periféricos que son considerados: lector de código de barras, caja de 
cobro, impresora de tickets, terminal de cobro con tarjetas bancaria e, incluso, al considerar la conectividad en red por manejo de multisucursales, o, en el sentido de máxima escalabilidad, por ejemplo, la necesidad de conectar el ERP con el "ecommerce”. Así como se estipula esta conexión con accesorios imprescindibles en las operaciones diarias, se presentan soluciones de $\mathrm{TI}$ disponibles en los dispositivos móviles diversos, tales como las herramientas digitales, de diversos aplicativos que coadyuven en algún área del proceso administrativo, que sirva tanto para gestión interna del conocimiento, como para mejorar la competitividad en el mercado en que opera.

3. En cuanto a la estrategia digital de mercadeo y ventas, el posicionamiento que permite un manejo adecuado de redes sociales, página web empresarial, más el apoyo del comercio electrónico, en los diferentes momentos (preventa, experiencia de compra, posventa y servicio y/o soporte técnico, incluso en atención al cliente, con una mesa de ayuda, chatbot o telemarketing y comunicación en tiempo real por las diversas plataformas de mensajería y/o teleconferencia) permitirán una competitividad mayor que genere valor, así también ofrezca métricas de la relación con clientes, e incluso, con proveedores como parte de la cadena de valor.

4. Se debe considerar la estimación financiera, misma que queda fuera del alcance del presente estudio, acerca de la inversión a realizar, la cual se justifica con los porcentajes obtenidos en relación con el impacto positivo en la competitividad de la MIPYME. Para esta estimación y presupuesto en caso formal, se propone como un proyecto de inversión alterno para buscar cubrir las necesidades básicas que permitan la viabilidad de este, en el entendido que, con un proyecto de inversión, elaborado con los estudios imprescindibles, se pueden encontrar opciones de financiamiento para este sector empresarial.

5. Cabe señalar que la capacitación en el manejo de las alternativas de $\mathrm{TI}$ quedará a consideración del empresario o área directiva, para que se imparta de acuerdo a las expectativas propias, ya sea solo al empresario, directivos o a personal operativo, en sus respectivas áreas en que se relacionen los perfiles de puesto. En este sentido, también se hace presente la relación de comunicación con el área de soporte técnico o posventa.

6. Por último, las consideraciones para proyectos de desarrollo de aplicativos de acuerdo con la solicitud del empresario o directivo, así como lo relativo a plataformas de comercio electrónico, redes sociales mesa de ayuda, y otros servicios, quedará sustentada en la parte diagnóstica siendo, sin embargo, un elemento alterno en cuanto la intervención contemplada para este método.

\section{CONCLUSIONES}

Esta investigación puede tener continuidad, si en alguno de los negocios incluidos en la muestra, se lleva a cabo un proyecto de intervención con base en la metodología propuesta de los 6 puntos clave de integración de las TI para incrementar la competitividad de las empresas, con ello se podrá evaluar si hubo un impulso y mejora en las empresas.

Por otro lado, como hemos observado, a pesar de tener un mundo completamente digital al alcance de 
cualquiera, aún existe una resistencia ante el cambio, detectado precisamente en la mayoría de las empresas analizadas. Curiosamente, se puede intuir por los resultados, que el uso y manejo de las $\mathrm{TI}$, así como de aquellos instrumentos que la rodean, se encuentra todavía en etapa de transición, qué quiere decir esto, que las personas que laboran en estos espacios, consideran que el tener una persona encargada de sus redes sociales y el marketing digital, así como de un catálogo para ventas en plataformas tecnológicas, aún es innecesario, por otro lado, los mismos dueños o encargados de los mismos, realizan estas acciones por su cuenta.

Para el momento que estamos viviendo, lo ideal que nuestros resultados hubieran arrojado, es que al menos un $80 \%$ de todas las empresas encuestadas utilizaran en su totalidad al menos un sistema ERP ya sea libre o de licencia, un catálogo digital y que a su vez, pudiera hacer ventas a través de estos medios. También cabe resaltar que tiene un impacto más allá de lo evidente, cuestión que sería importante hacerle saber a todas aquellas organizaciones dispuestas a migrar a lo digital, desde reducción de costos en ámbito de crecer con menos recursos, así como llegar a un público más amplio sin necesidad de pertenecer a la misma ciudad, incluso.

Continuar con el esquema planteado en el presente artículo, con base en los 6 pasos establecidos, ayudará a mejorar la participación potencial de las empresas en el medio electrónico y además, alentará a que aquellos que aún no se suman a esta causa y una vez que lo hagan, cuenten con una estructura sólida, en la cual vale la pena adaptarse y que a la larga ofrecerá grandes beneficios.
La expectativa a largo plazo, será que todos los negocios, organizaciones y demás, pertenezcan al ámbito digital, es decir que el comercio electrónico sea la nueva cotidianidad, la previsión estima que aproximadamente en 15 o 20 años, si no es que menos, queden solo un par de establecimientos físicos, o simplemente aquellos que sea estrictamente necesario, por lo cual, estamos en el momento perfecto para ir incursionando y explorando las nuevas alternativas de vanguardia, por último, se recomienda acercarse a analizar las nuevas tendencias en países de mayor desarrollo, Europa, se ha caracterizado por apostar sus esfuerzos en este tópico.

Finalmente, si logramos crear nuevas organizaciones electrónicas a partir de este momento y antes que nadie, supone una ventaja mayoritaria sobre todos los competidores, siendo un nicho primario para su crecimiento oportuno y exponencial, coadyuvando a la integración de un mundo globalizado.

\section{REFERENCIAS}

Andreu, R., Ricart, J., y Valor, J. (I99I). Estrategia y sistema de información. McGraw-Hill. Interamericana de España, Madrid.

Arévalo, J. A. (2007). Gestión de la Información, Gestión de Contenidos y Conocimiento. II Jornadas de Trabajo del Grupo SIOU

Argüelles Ma, L. A., Quijano García, R. A., Fajardo, M. J., Medina Blum, F., y Cruz Mora, C. E. (2018). El Endeudamiento como Indicador de Rentabilidad Financiera en las Mipymes Turísticas de Campeche (2018). Revista Internacional Administracion \& Finanzas, II (I), 39-5I.

Arner, D., Barberis, J., y Buckley, R. (20I5). The evolution of Fintech: A new post-crisis paradigm. Geo. J. Int'l L., 47, I27I.

Arnold Cathalifaud, M., Osorio, F. (1998). Introducción a los Conceptos Básicos de la Teoría General de Sistemas. Cinta de Moebio, (3). 
Bayraktar, B.A. (1990). On the concepts of technology and management of technology. Proceedings of the Second International Conference on Management of Technology (IAMOT, 1990), Khalil, T.M. y

Bayraktar, B.A, eds.: II6I-II75.

Blázquez, A. (2013). Un modelo de pensamiento estratégico para favorecer la gestión del cambio en las organizaciones. AD-minister (23).

Chacón, G. (2007). La Contabilidad de Costos, los Sistemas de Control de Gestión y la Rentabilidad Empresarial. Actualidad Contable Faces, I0(I5).

Confederación de Cámaras Nacionales de Comercio, Servicios y Turismo [CONCANACO SERVYTUR]. (20I8). Indicadores Veracruz. Recuperado de http://www.concanaco.com.mx/documentos/indi cadoresestados/Veracruz.pdf

Contreras Montellano, Ó. F., y García Fuentes, M. (2019). Pequeñas y medianas empresas tecnológicas en México: distribución regional e inserción en cadenas globales de valor. Región y Sociedad, 31, https://doi.org//0.22198/rys2019/31//234

Daza Izquierdo, J. (2016). Crecimiento y rentabilidad empresarial en el sector industrial brasileño. Contaduría y administración, 6I (2), 266-282. https://dx.doi.org/10.1016/j.cya.2015.12.001

Dessler, G. (2009). Administración de recursos humanos (II. a ed.). México: Pearson

García Garnica, A., y Taboada Ibarra, E. L. (20/2). Teoría de la empresa: las propuestas de Coase, Alchian y Demsetz, Williamson, Penrose y Nooteboom. Economía: teoría y práctica, (36), 942.

Hernández Palma, H., Barrios Parejo, I., y Martínez Sierra, D. (20/8). Gestión de la calidad: elemento clave para el desarrollo de las organizaciones. Criterio Libre, 16(28), 169-185.

https://doi.org/10.18041/19000642/criteriolibre.2018v16n28.2130

Ibarra, M. A., González, L. A. y Demuner, M. del R. (2017). Competitividad empresarial de las pequeñas y medianas empresas manufactureras de Baja California. Estudios Fronterizos, 18(35), I07-130,

https://doi.org// 0.21670/ref.2017.35.a06

Instituto Nacional de Estadística y Geografía [INEGI] (2018). Sistema de Clasificación Industrial de América del Norte, México SCIAN 2018.
Instituto Nacional de Estadística y Geografía [INEGI]. (20I7). Cuéntame. Información por entidad. Recuperado de: http://cuentame.inegi.org.mx/monografias/inform acion/ver/economia/default.aspx?tema $=$ me $\&$ e $=30$

Instituto Nacional de Estadística y Geografía [INEGI]. (20I8). Encuesta Nacional sobre Productividad y Competitividad de las Micro, Pequeñas y Medianas Empresas (ENAPROCE) 2018, Recuperado de: https://www.inegi.org.mx/contenidos/programas/ enaproce/2018/doc/EN APROCE20 I8Pres.pdf

Laudon, K. C. y Laudon, J. P. (1996). Administración de los Sistemas de Información, Prentice Hall, México

Ley para el desarrollo de la competitividad de la micro, pequeña y mediana empresa de 2019. 13 de agosto de 2019.

Molloy, S., Schwenk, C. R. (1995). The Effects of Information Technology on Strategic Decision Making, Journal of Management Studies, 32 (3), 283-3II.

Morales Flores, E. (2004). La gestión y los gestores de la información. Bibliodocencia 4 (4).

Morales González, M. A., Pech Várguez, J. L. (2000). Competitividad y estrategia: el enfoque de las competencias esenciales y el enfoque basado en los recursos. Revista contaduría y administración, 197, 47-63.

Organización de Cooperación y Desarrollo Económico [OCDE]. (2005). Manual de Oslo: Guía para la recogida e interpretación de datos sobre innovación. $3^{\mathrm{a}}$ ed., Oficina de Estadísticas de las Comunidades Europeas, París: OCDE, 194p.

Porter, M. (1 986). Ventaja Competitiva. Nueva York, Estados Unidos: Free Press.

Quispe-Otacoma, A. L., Padilla-Martínez, M. P., TelotGonzález, J. A., y Nogueira-Rivera, D. (20I7). Tecnologías de información y comunicación en la gestión empresarial de pymes comerciales. Ingeniería Industrial, 38(I), 8I-92.

Ramírez Galaviz, A. (2017). Los factores de la competitividad en las MiPymes en México. Revista Iberoamericana de Producción Académica y Gestión Educativa, 4 (17), I-I2.

Reyes Echeagaray, D. A. (coord.), Bribiesca Correa, G., Carrillo López, V. H., Corona Cabrera, A., Cruz Quiroz, R. A., Ramírez Munive, Y. A., Ramírez Chavero, M. O., Reyes Echeagaray, D. A., y Torres Garibay, R. (20I6). Tecnologías de Información y Comunicación en las Organizaciones, UNAM, México. 
Rivas Tovar, L. A. (2009). Evolución de la teoría de la organización. Universidad \& Empresa, I I ( 77), I I32.

Ros García, J. (1993). Auge de los sistemas de Información y Documentación en las organizaciones. Cuadernos de documentación multimedia, 2.

Saavedra García, M. L. (20I2). Una propuesta para la determinación de la competitividad en la pyme latinoamericana. Pensamiento \& Gestión, (33), 93124.

Shu, C. (20I4). Dwolla fined $\$ 100,000$ for misrepresenting its data-security practices. Recuperado de: https://techcrunch.com/2016/03/02/dwolla-fined100000 -for-misrepresenting-its-data-securitypractices/\#: :text=Online\%20payment\%20proce ssing\%20startup\%20Dwolla,of\%20its\%20data\%2D security\%20practices.

WEFORUM. (2016) ¿Qué es la competitividad?

Recuperado de:

https://es.weforum.org/agenda/2016/10/que-es-

la-competitividad/ 